\title{
Multimodality Imaging Approach for Combined Central Retinal Vein and Artery Occlusion: The Role of Optical Coherence Tomography Angiography
}

\author{
Marta Díez-Sotelo ${ }^{a, b} \quad$ Maximino Abraldes ${ }^{a-c} \quad$ Francisco Gómez-Ulla $^{b, c}$ \\ aDepartment of Ophthalmology, Complejo Hospitalario Universitario de Santiago de \\ Compostela, Santiago de Compostela, Spain; bUniversity of Santiago de Compostela, \\ Santiago de Compostela, Spain; 'Instituto Oftalmológico Gómez-Ulla, Santiago de \\ Compostela, Spain
}

\section{Keywords}

Optical coherence tomography angiography $\cdot$ Retinal vein occlusion $\cdot$ Retinal artery occlusion - Fluorescein angiography - Imaging retina

\begin{abstract}
Combined central retinal artery and vein occlusion is an uncommon vascular pathology that can cause severe and permanent visual impairment. Optical coherence tomography angiography (OCTA) is a newly available, noninvasive imaging technique that can potentially improve understanding of the structural and vascular implications and prognosis of this infrequent pathology. The present report describes the principal clinical findings in a case of combined central retinal artery and vein occlusion, as detected by the different imaging modalities available in a tertiary referral hospital. OCTA wide-field montage images identified an extensive area of nonperfusion on the macula with involvement of the entire retina at nearly $360^{\circ}$. We observed the most severe nonperfusion in the deep capillary plexus, while perfusion of the choriocapillaris was unaffected. Meanwhile, fluorescein angiography (FA) findings revealed a delay in perfusion rate with marked nonperfusion areas in the peripheral retina at $360^{\circ}$. We identified that the wide-field OCTA montage permitted visualization of a similar or wider peripheral retinal
\end{abstract}




\section{Case Reports in Ophthalmology}

area compared with FA. Therefore, OCTA is potentially useful for assessment of the global retinal nonperfusion status at baseline and during follow-up, with the added advantage of being a noninvasive technique.

\section{Introduction}

Retinal vein occlusion (RVO) is the second most frequent cause of retinal vascular disease after diabetic retinopathy [1]. Its most severe manifestation is ischemic central RVO (CRVO), which causes visual impairment and a risk of neovascularization. Combined central retinal artery occlusion (CRAO) and CRVO is an uncommon vascular pathology generally associated with systemic disease such as immunological illnesses, malignancies, and coagulopathies [2].

Fluorescein angiography (FA) has remained the gold standard for visualization of the vascular pathologies of retinal diseases until the advent of optical coherence tomography angiography (OCTA), a novel and noninvasive technique based on OCT technology. OCTA is not a substitute, but rather a complementary technique, to previously available imaging techniques. OCTA enables independent visualization of not only the superficial capillary plexus (SCP), but also the deep capillary plexus (DCP) and the choriocapillaris, allowing a better understanding of retinal vascular injuries and pathologies.

The aim of the present report was to describe and illustrate the principal clinical findings in a patient diagnosed with combined CRVO and CRAO. To our knowledge, this report is the first to directly compare FA and wide-field OCTA montage findings in this uncommon pathology.

\section{Case Report}

An 82-year-old man presented with a 2-week history of sudden painless permanent blurred vision in his right eye (OD), without any systemic symptoms such as headache, polymyalgia rheumatic, or jaw claudication that would be indicative of giant cell arteritis. The patient did not report vision loss or other alterations in his left eye (OS). He had a history of arterial hypertension, dyslipidemia, and atrial fibrillation treated with antihypertensive drugs and statins, and was a former smoker. The patient received treatment with acenocoumarol some months prior to the onset of symptoms, but at the time of the first visit, and by his own decision, the patient was not taking this treatment. He reported no surgical or medical ophthalmological history.

The patient underwent a complete ophthalmic examination at baseline and at the 3-and 6-month follow-up visits, including best-corrected visual acuity (BCVA) measurement with the Early Treatment Diabetic Retinopathy Study (ETDRS) chart, intraocular pressure (IOP) measurement, anterior segment examination, funduscopic study, gonioscopy, OCT, and OCTA with a Swept Source OCT Triton (Topcon Corp., Tokyo, Japan) performing $3 \times 3$ and $6 \times 6 \mathrm{~mm}$ captures centered on the fovea. Wide-field OCTA montages were also performed with peripheral $6 \times 6$ and $9 \times 9 \mathrm{~mm}$ images captured at baseline and at the 3- and 6-month follow-up visits. Adobe Photoshop CC 2018 (Adobe Systems, San Jose, CA, USA) was used to manually create the montages. FA (Spectralis Heidelberg Engineering, Heidelberg, Germany) was also performed at baseline and at the 6-month follow-up visit.

At the first visit, the BCVA was hand motion in the OD and 20/32 in the OS; IOP was 14 and $12 \mathrm{~mm} \mathrm{Hg}$, respectively. Slit lamp examination detected a bilateral nuclear cataract. 


\section{Case Reports in Ophthalmology}

Funduscopic study revealed hypertensive retinopathy grade 1 and disperse drusen in the OS. The retina of the right eye had a congested appearance with papillary edema, and intraretinal and widespread flame-shaped hemorrhages in all four quadrants, prompting a diagnosis of CRVO. Baseline OCT line HD and macular OCTA findings of $3 \times 3$ and $6 \times 6 \mathrm{~mm}$ captures centered on the fovea; initial FA findings are shown and explained in Figure 1.

Anti-VEGF therapy was the first-line treatment for the patient. He received an initial loading dose of 3-monthly intravitreal injections of aflibercept, followed by a pro re nata regimen over a 6-month period.

At the 3-month funduscopic examination, resorption of the hemorrhages had improved visualization of the vascular structures. Fundus color photography identified more scattered flame-shaped hemorrhages than at baseline, without papillary edema, and extensive emboli on the principal temporal retinal arteries. The latter presented a funduscopic appearance of platelet-fibrin emboli due to their duller white-gray coloration and their stretched arrangement in the arterial lumen. OCT line HD identified more evident inner-layer hyperreflectivity and an important atrophy of inner and outer layers, without retinal edema. Red-free filter photography revealed hyperreflective images corresponding to the emboli on the fundus color photography, confirming the final diagnosis of combined CRVO and CRAO. The absence of whitish coloration of nonperfused retina can be explained by the time of evolution since the diagnosis, until the hemorrhages disappeared, as this sign usually lasts a few weeks. The macular OCT and OCTA findings of the 3-month follow-up visit are shown and described in Figure 2. The wide-field OCTA montage at the 3-month follow-up visit indicated extensive macular and peripheral nonperfusion areas (Fig. 2).

At the 3-month follow-up visit, the BCVA was counting fingers in the OD, which remained unchanged at the 6-month follow-up. In the OS, an IOP of $28 \mathrm{~mm} \mathrm{Hg}$ at the 3-month examination prompted treatment with a topical prostaglandin analog. The OD with CRVO and CRAO had normal-range IOP. At the 6-month follow-up visit, the patient had not developed rubeosis iridis on the OD, and gonioscopy demonstrated an open angle without signs of angle neovascularization. The funduscopic examination remained similar to that described at the 3-month follow-up visit, without signs of retinal neovascularization. However, some slight differences in the location of the emboli could be observed, which supports the diagnosis of platelet-fibrin emboli causing CRAO. Autofluorescence photography showed hyperautofluorescent images corresponding to emboli observed in the fundus color photography. Macular OCT and OCTA findings, as well as the final FA and wide-field OCTA montages performed at the 6-month follow-up visit, are shown and described in Figure 3.

\section{Discussion}

OCTA is a novel, noninvasive imaging method based on the flow rate of erythrocytes moving above a detection rate threshold through the blood vessel lumen. OCTA uses algorithms to measure the variation in reflected OCT signal amplitude between consecutive cross-sectional B-scans, generating high-quality angiograms of the retina and choroid [3]. SS-OCT Triton was used to obtain OCTA images. This instrument has an A-scan rate of 100,000 scans/s, and uses OCTARA ${ }^{\odot}$ detection software, which is more sensitive in the detection of low or slow blood flow than other OCTA methods and is the only commercially available SS-OCTA with a 1,050nm light source [4]. 


\section{Case Reports in Ophthalmology}

In the past 4 years, OCTA has been used to identify previously unknown pathophysiological features of retinal vascular diseases such as branch RVO (BRVO) [5-7], CRVO [5, 7, 8], and RAO $[9,10]$.

Prior findings have suggested that the DCP is most significantly damaged by RVO [5, 11], necessitating independent evaluation of each plexus. OCTA provides stratigraphic vascular details that have not previously been described with standard FA, which may assist assessments of the extent of retinal nonperfusion areas at baseline and during follow-up. Furthermore, this can provide information than can help predict responses to anti-VEGF treatment and vascular prognosis in vascular diseases. In addition, OCTA technology has the added advantages of being noninvasive and less time-consuming.

Chung and Li [12] reported a case of BRVO and performed a wide-field montage before and after anti-VEGF treatment, which yielded a visualization area of $8 \times 8 \mathrm{~mm}$. This is smaller than the area achieved in the second and third montages in our case.

Kaya et al. [13] reported a case of cilio-RAO combined with CRVO, comparing macular OCTA findings with FA images 10 months after treatment with a single intravitreal injection of dexamethasone. In such a chronic phase of the disease, FA showed normal filling of the cilioretinal artery with correct perfusion of the retinal capillary network on the macula. Conversely, OCTA identified nonperfusion areas in both the SCP and DCP, demonstrating its superiority over FA in evaluating the vascular status of the macula in this disease. In our study, we compared FA and OCTA findings not only in the chronic phase but also at baseline. This allowed us to perform suitable imaging of the macula and detect the presence of wide areas of macular ischemia on the SCP and suspected nonperfused areas on the DCP even at the first visit, and to predict the vascular evolution of the disease and its response to treatment. Furthermore, Kaya et al. [13] did not study the peripheral findings on OCTA, as we did.

Wu et al. [14] presented an interesting case of combined CRVO and CRAO that occurred 9 days following cataract surgery with retrobulbar anesthesia, reporting major OCT and OCTA macular findings in this rare pathology for the first time. As reported here, the authors observed the absence of perfusion in both capillary layers on OCTA, with minimal involvement of choriocapillaris. OCT revealed inner-layer hyperreflectivity of the retina and structural alteration of the external layers. Unfortunately, they did not report OCT, OCTA, or FA follow-up findings. Moreover, they did not include peripheral OCTA montages nor did they compare their findings with FA, so no information regarding peripheral perfusion was provided. As described in these prior studies, and confirmed in our report, the choriocapillaris layer appears to be unaffected in combined CRVO and CRAO.

The present study evaluated a case of combined CRVO and CRAO not only with macular OCTA images but also with wide-field OCTA montages to compare the nonperfusion status with FA.

Our patient presented poor fixation at the baseline visit. This enabled us to perform OCTA in the macular and papillary area, but not in the peripheral retina. However, these images provided more information regarding the posterior pole nonperfusion status in the SCP than did FA, due to limited visualization of retinal perfusion with FA caused by the screen effect of the hemorrhages and the contrast leakage. Kashani et al. [15] demonstrated that in OCTA, retinal perfusion can only be assessed in the retinal layers superficial to those involved with intraretinal hemorrhages, as the hemorrhages block the OCTA signal to some extent and cause disruption of the capillary network with no flow signal. Furthermore, intraretinal hemorrhages in CRVO are detected more frequently in DCP than in SCP [15]. Therefore, OCTA allows us to reliably visualize the SCP but not the DCP in the presence of intraretinal hemorrhages. Although neither technique could initially provide us with information about the DCP due to 
hemorrhages, OCTA gave us at least the same information about macular SCP as FA, with a higher level of capillary resolution, which is difficult to achieve using standard FA.

At the 6-month post-treatment visit, the wide-field OCTA montage permitted visualization of a similar or wider peripheral area than did the FA image. Although OCTA does not provide dynamic information about vascular flow, the present study suggests that it is a useful tool for assessment of the global nonperfusion status of the retina at baseline and during follow-up. Furthermore, OCTA can be performed at every visit and in every patient.

The main limitation of this technique is that it is time-consuming to obtain the wide-field OCTA montage; therefore, the development of automated montage software should be considered because it would be less time-consuming and easier to implement in clinical practice.

To our knowledge, this is the first report to describe the main macular and peripheral OCTA findings of a case of combined CRVO and CRAO, in addition to complete imaging findings at baseline and at the 3- and 6-month follow-up visits, including global perfusion status visualization with SS-OCTA wide-field montages. This report contributes to the understanding of the structural and vascular implications and prognosis of this uncommon disease, without the invasiveness of classic angiography techniques.

\section{Statement of Ethics}

This case study was performed in the Ophthalmology Department of the Clinical University Hospital of Santiago de Compostela, Spain. It adhered to the tenets of the Declaration of Helsinki and was approved by the Research Ethics Committee of the Autonomous Community of Galicia (CAEIC), the committee attached to the regional government, and the General Technical Secretariat of the Health Ministry of the Xunta de Galicia (protocol code: MAL-OCTA2018-01). The patient gave written informed consent to publish this case, including publication of images.

\section{Disclosure Statement}

The authors have no conflicts of interest to declare.

\section{Funding Sources}

This work was supported in part by the Mutua Madrileña project (Ref. 2017/365).

\section{Author Contributions}

M.D.-S.: methodology, investigation, resources, data curation, and writing (original draft preparation). M.A. and M.D.-S.: formal analysis. M.A. and F.G.-U.: conceptualization, writing (review and editing), and supervision. 


\section{Case Reports in Ophthalmology}

\section{References}

1 Klein R, Klein BE, Moss SE, Meuer SM. The epidemiology of retinal vein occlusion: the Beaver Dam Eye Study. Trans Am Ophthalmol Soc. 2000;98:133-41.

2 Schmidt D. Comorbidities in combined retinal artery and vein occlusions. Eur J Med Res. 2013 Aug;18(1):27.

3 Nobre Cardoso J, Keane PA, Sim DA, Bradley P, Agrawal R, Addison PK, et al. Systematic evaluation of optical coherence tomography angiography in retinal vein occlusion. Am J Ophthalmol. 2016 Mar;163:93-107.e6.

4 Stanga PE, Tsamis E, Papayannis A, Stringa F, Cole T, Jalil A. Swept-Source Optical Coherence Tomography Angio $^{\mathrm{TM}}$ (Topcon Corp, Japan): technology review. Dev Ophthalmol. 2016;56:13-7.

5 Coscas F, Glacet-Bernard A, Miere A, Caillaux V, Uzzan J, Lupidi M, et al. Optical coherence tomography angiography in retinal vein occlusion: evaluation of superficial and deep capillary plexa. Am J Ophthalmol. 2016 Jan;161:160-71.e1.

6 Samara WA, Shahlaee A, Sridhar J, Khan MA, Ho AC, Hsu J. Quantitative optical coherence tomography angiography features and visual function in eyes with branch retinal vein occlusion. Am J Ophthalmol. 2016 Jun;166:76-83.

7 Mastropasqua R, Toto L, Di Antonio L, Borrelli E, Senatore A, Di Nicola M, et al. Optical coherence tomography angiography microvascular findings in macular edema due to central and branch retinal vein occlusions. Sci Rep. 2017 Jan;7(1):40763.

8 Winegarner A, Wakabayashi T, Hara-Ueno C, Sato T, Busch C, Fukushima Y, et al. Retinal microvasculature and visual acuity after intravitreal aflibercept in eyes with central retinal vein occlusion: an optical coherence tomography angiography study. Retina. 2018 Oct;38(10):2067-72.

9 Baumal CR. Optical coherence tomography angiography of retinal artery occlusion. Dev Ophthalmol. 2016;56:122-31.

10 Hwang CK, Kolomeyer AM, Brucker AJ. Optical coherence tomography angiography of a central retinal artery occlusion before and after anterior chamber paracentesis. Ophthalmology. 2017 May;124(5):608.

11 Kang JW, Yoo R, Jo YH, Kim HC. Correlation of microvascular structures on optical coherence tomography angiography with visual acuity in retinal vein occlusion. Retina. 2017 Sep;37(9):1700-9.

12 Chung CY, Li KK. Optical coherence tomography angiography wide-field montage in branch retinal vein occlusion before and after anti-vascular endothelial-derived growth factor injection. Int Ophthalmol. 2018 Jun;38(3):1305-7.

13 Kaya M, Ozturk T, Ayhan Z, Kocak N, Kaynak S. Cilioretinal artery occlusion combined with central retinal vein occlusion: what is the best imaging modality for the follow-up? Case Rep Ophthalmol Med. 2017;2017:9032576.

14 Wu SC, Villegas VM, Kovach JL. Optical coherence tomography angiography of combined central retinal artery and vein occlusion. Case Rep Ophthalmol Med. 2018 Feb;2018:4342158.

15 Kashani AH, Lee SY, Moshfeghi A, Durbin MK, Puliafito CA. Optical coherence tomography angiography of retinal venous occlusion. Retina. 2015 Nov;35(11):2323-31. 


\section{Case Reports in Ophthalmology}
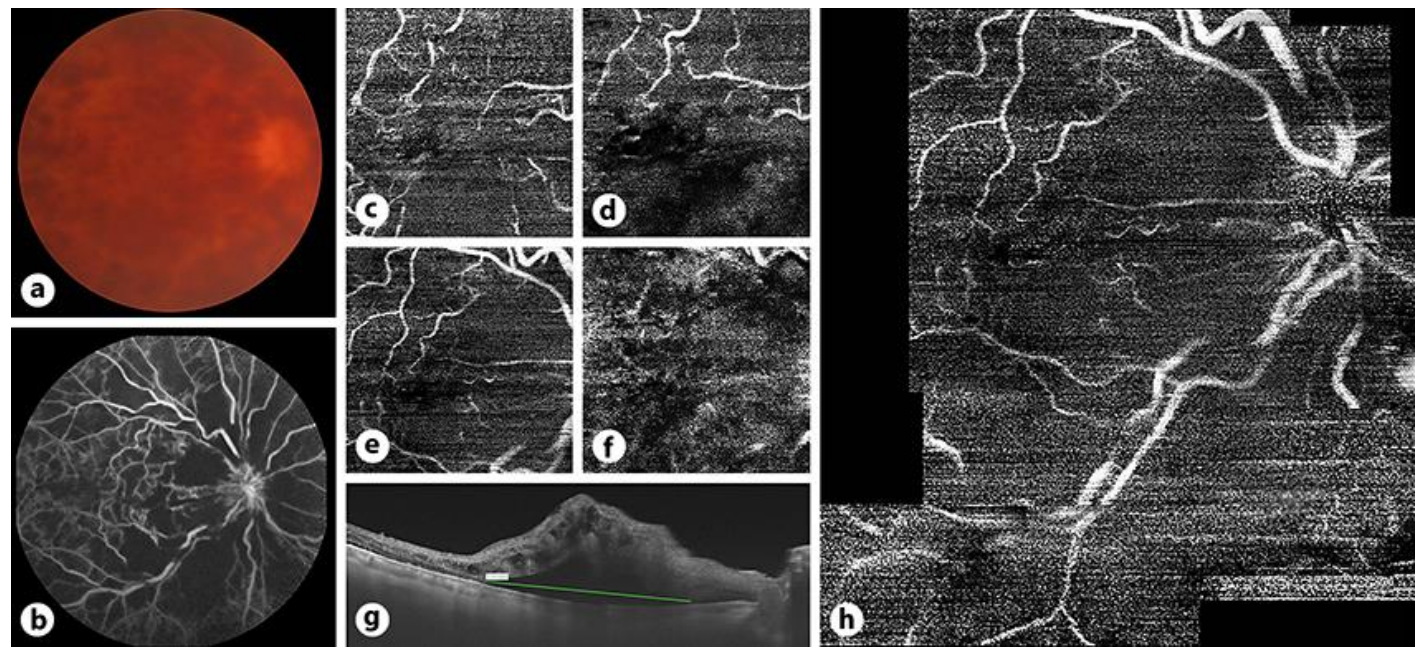

Fig. 1. Baseline fundus color photography (a) and fluorescein angiography (FA) (b). Optical coherence tomography angiography (OCTA) of the superficial capillary plexus (SCP) (c) and deep capillary plexus (DCP) (d) in the $3 \times 3 \mathrm{~mm}$ area centered on the fovea, and of the SCP (e) and DCP (f) in the $6 \times 6 \mathrm{~mm}$ area centered on the fovea. $\mathbf{g}$ Baseline macular OCT line HD. $\mathbf{h}$ OCTA montage of the posterior pole. FA could not define the presence of macular or peripheral ischemia due to the screen effect from hemorrhage blockage. OCTA revealed a severe macular nonperfusion area on the $3 \times 3$ and $6 \times 6 \mathrm{~mm}$ images. Despite the retinal hemorrhages, OCTA provided independent information about SCP and DCP. However, DCP was mainly affected by intraretinal hemorrhages, thus the lower patched reflectivity shown in DCP macular images could correspond to intraretinal hemorrhages rather than nonperfused areas. Baseline OCT line HD revealed intraretinal hemorrhages and a 1,108- $\mu$ m-thick central macular edema with a 5,173- $\mu \mathrm{m}$-length neurosensorial detachment and a subtle hyperreflectivity of the inner macular layers that went unnoticed during the first examination. The posterior pole OCTA montage shows motion artifacts, which were caused by poor fixation of the patient. 


\section{Case Reports in Ophthalmology}
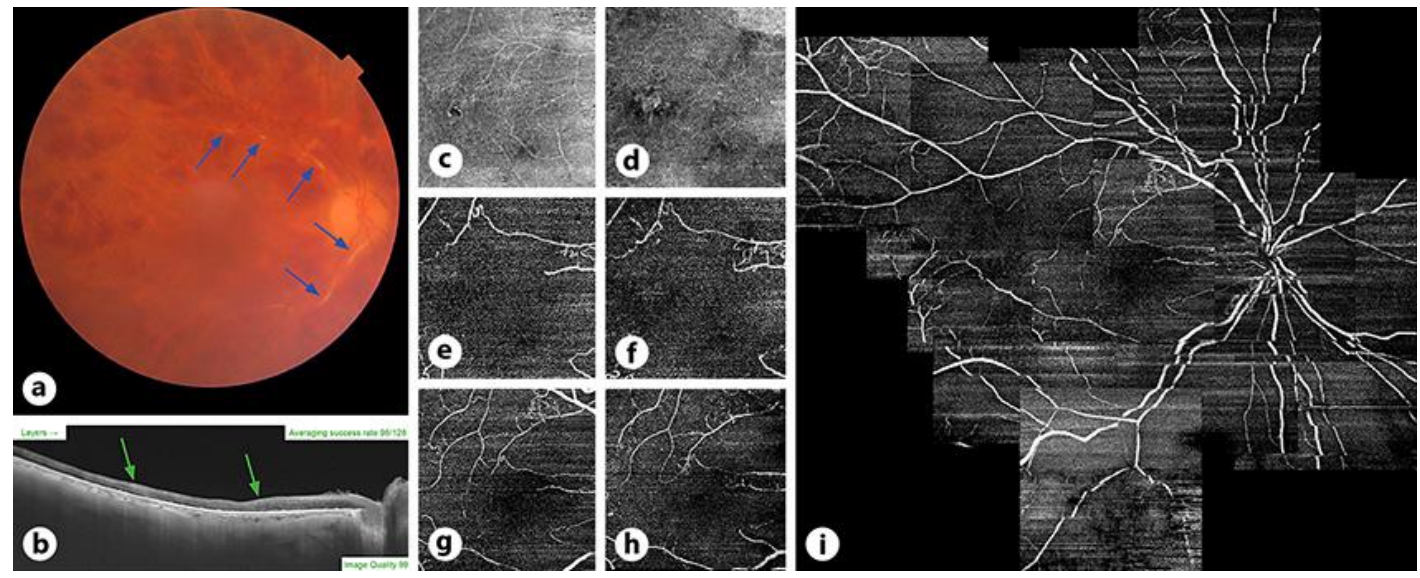

Fig. 2. Three-month follow-up fundus color photography (a), macular OCT line HD (b), and en face $3 \times 3$ mm superficial capillary plexus (SCP) (c) and deep capillary plexus (DCP) (d) images. OCTA images of the SCP (e) and DCP (f) in the $3 \times 3 \mathrm{~mm}$ area centered on the fovea, and SCP (g) and DCP (h) in the $6 \times 6 \mathrm{~mm}$ area centered on the fovea. i Wide-field OCTA montage ( $6 \times 6 \mathrm{~mm}$ images). Fundus color photography revealed scattered flame-shaped hemorrhages without papillary edema and extensive white-gray images within the arterial lumen with the appearance of platelet-fibrin emboli on the principal temporal retinal arteries (blue arrows). OCT line HD revealed an evident inner-layer hyperreflectivity (green arrows) and an important atrophy of external layers, without retinal edema. OCTA images indicated the nonperfusion status of the macula, which was present in both the SCP and DCP. The wide-field OCTA montage revealed extensive macular and peripheral nonperfusion areas. 


\section{Case Reports in Ophthalmology}
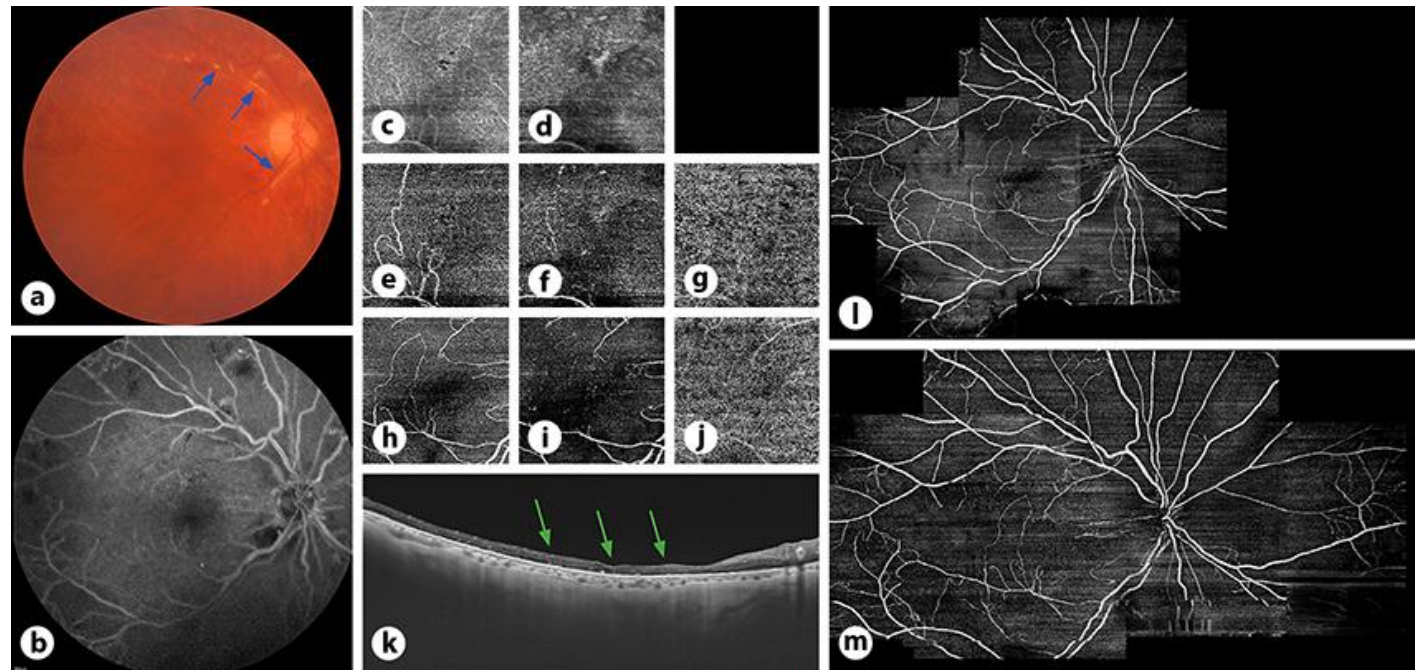

Fig. 3. Six-month follow-up fundus color photography (a) and fluorescein angiography (FA) (b). En face $3 \times 3 \mathrm{~mm}$ superficial capillary plexus (SCP) (c) and deep capillary plexus (DCP) (d) images. OCTA of the SCP (e) and DCP (f) in the $3 \times 3 \mathrm{~mm}$ area centered on the fovea, and SCP (h) and DCP (i) in the $6 \times 6 \mathrm{~mm}$ area centered on the fovea. OCTA of the choriocapillaris in the $3 \times 3(\mathrm{~g})$ and $6 \times 6(\mathrm{j}) \mathrm{mm}$ areas centered on the fovea. $\mathbf{k}$ Six-month follow-up macular OCT line HD. Wide-field OCTA montage made with $6 \times 6 \mathrm{~mm}$ (I) and $9 \times 9 \mathrm{~mm}(\mathrm{~m})$ images. Fundus color photography revealed extensive emboli on the principal temporal retinal arteries (blue arrows). Compared to the 3-month follow-up fundus color photography, some differences in the location of the emboli can be observed. FA images at the end of follow-up revealed a delayed perfusion rate with localized thinning of the arterial vascular lumen close to the papilla and marked nonperfusion areas in the peripheral retina at $360^{\circ}$, without evidence of retinal neovascularization. In such a chronic phase of the disease, the arterioles might be reperfused, so no stops in the vascular column could be observed. FA does not show any vascular leakage or staining. Macular OCTA images revealed severe involvement of the deep capillary plexus, with no change in perfusion of the choriocapillaris. OCT line HD showed an important atrophy of the external layers, without retinal edema (green arrows). The OCTA wide-field montage images demonstrated an extensive nonperfusion area on the macula with involvement of the entire retina at nearly $360^{\circ}$. Principal temporal arteries are entirely represented in OCTA, thus we assume that these arteries are reperfused. The OCTA montages allowed visualization of at least the same or wider nonperfusion peripheral areas compared with FA. 